CUBO A Mathematical Journal

Vol.14, No 01 , (119-125). March 2012

\title{
Majorization for certain classes of analytic functions defined by a new operator
}

\author{
E. A. Eljamal and M. Darus \\ School of Mathematical Sciences, \\ Faculty of Science and Technology, \\ Universiti Kebangsaan Malaysia, \\ Bangi 43600 Selangor D. Ehsan, Malaysia. \\ email: n-ebtisam@yahoo.com, maslina@ukm.my
}

\begin{abstract}
In the present paper, we investigate the majorization properties for certain classes of multivalent analytic functions defined by a new operator. Moreover, we pointed out some new and known consequences of our main result.
\end{abstract}

\section{RESUMEN}

En el presente artículo, investigamos las propiedades de mayorización para ciertas clases de funciones analíticas multivalentes definidas por un nuevo operador. Además, resaltamos algunas consecuencias -nuevas y conocidas- de nuestro resultado princresultado.

Keywords and Phrases: Majorization properties, multivalent functions, Ruscheweyh derivative operator, Hadamard product.

2010 AMS Mathematics Subject Classification: 30C45. 


\section{Introduction}

Let $f$ and $g$ be analytic in the open unit disk $U=\{z: z \in \mathbb{C},|z|<1\}$. We say that $f$ is majorized by $\mathrm{g}$ in $\mathrm{U}$ and write

$$
\mathrm{f}(z) \ll \mathrm{g}(z) \quad(z \in \mathrm{U})
$$

if there exists a function $\varphi$, analytic in $\mathrm{U}$ such that

$$
|\varphi(z)| \leq 1 \quad \text { and } \quad f(z)=\varphi(z) g(z) \quad(z \in \mathcal{U}) .
$$

It maybe noted here that (1.1) is closely related to the concept of quasi-subordination between analytic functions. Let $A_{p}$ denote the class of functions of the form

$$
f(z)=z^{p}+\sum_{k=p+1}^{\infty} a_{k} z^{k},(p \in \mathbb{N}=\{1,2, \ldots\})
$$

which are analytic and multivalent in the open unit disk $U$. In particular, if $p=1$, then $A_{1}=A$. For functions $f_{j} \in A_{p}$ given by

$$
f_{j}(z)=z^{p}+\sum_{k=p+1}^{\infty} a_{k, j} z^{k},(j=1,2 ; p \in \mathbb{N}),
$$

we define the Hadamard product or convolution of two functions $f_{1}$ and $f_{2}$ by

$$
f_{1} * f_{2}(z)=z^{p}+\sum_{k=p+1}^{\infty} a_{k_{1}}, a_{k_{2}} z^{k}=\left(f_{2} * f_{1}\right)(z) .
$$

Definition 1.1. Let the function $\mathrm{f}$ be in the class $A_{\mathrm{p}}$. Ruscheweyh derivative operator is given by

$$
\mathrm{R}^{\mathrm{n}}=z^{\mathrm{p}}+\sum_{k=\mathrm{p}+1}^{\infty} \mathrm{C}(\mathrm{k}, \mathrm{n}) \mathrm{a}_{\mathrm{k}} z^{\mathrm{k}} .
$$

Next we define the following differential operator,

$$
\begin{gathered}
D^{\mathcal{O}}=f(z)=z^{p}+\sum_{k=p+1}^{\infty} a_{k} z^{k} \\
D_{n, \lambda_{1}, \lambda_{2}, p}^{1}=D^{0} f(z) \frac{p-p \lambda_{1}+\lambda_{2}(k-p)}{p+\lambda_{2}(k-p)}+\left(D^{0} f(z)\right)^{\prime} \frac{z \lambda_{1}}{p+\lambda_{2}(k-p)} \\
=z^{p}+\sum_{k=p+1}^{\infty}\left[\frac{p+\left(\lambda_{1}+\lambda_{2}\right)(k-p)}{p+\lambda_{2}(k-p)}\right] a_{k} z^{k},
\end{gathered}
$$

and

$$
D_{n, \lambda_{1}, \lambda_{2}, p}^{2}=D_{n, \lambda_{1}, \lambda_{2}, p}^{1} f(z) \frac{p-p \lambda_{1}+\lambda_{2}(k-p)}{p+\lambda_{2}(k-p)}+\left(D_{n, \lambda_{1}, \lambda_{2}, p}^{1} f(z)\right)^{\prime} \frac{z \lambda_{1}}{p+\lambda_{2}(k-p)}
$$




$$
=z^{p}+\Sigma_{k=p+1}^{\infty}\left[\frac{p+\left(\lambda_{1}+\lambda_{2}\right)(k-p)}{p+\lambda_{2}(k-p)}\right]^{2} a^{k} z^{k}
$$

In general,

$$
D_{n, \lambda_{1}, \lambda_{2}, p}^{m} f(z)=D\left(D^{n-1} f(z)=z^{p}+\Sigma_{k=p+1}^{\infty}\left[\frac{p+\left(\lambda_{1}+\lambda_{2}\right)(k-p)}{p+\lambda_{2}(k-p)}\right]^{m} a_{k} z^{k}\right.
$$

where ( $\left.\mathrm{m}, \mathrm{n} \in \mathbb{N}_{0}=\mathbb{N} \cup\{0\}, \lambda_{2} \geq \lambda_{1} \geq 0\right)$. By applying convolution product on (1.6) and (1.7) we have the following operator

$$
D_{n, \lambda_{1}, \lambda_{2}, p}^{m} f(z)=z^{p}+\Sigma_{k=p+1}^{\infty}\left[\frac{p+\left(\lambda_{1}+\lambda_{2}\right)(k-p)}{p+\lambda_{2}(k-p)}\right]^{m} C(k, n) a_{k} z^{k}
$$

where $\mathrm{C}(\mathrm{k}, \mathrm{n})=\frac{\Gamma(\mathrm{k}+\mathrm{n})}{\Gamma(\mathrm{k})}$.

Moreover, for $m, n \in N_{0}, \quad \lambda_{2} \geq \lambda_{1} \geq 0$

$$
\left(p+\lambda_{2}(k-p)\right) D_{\lambda_{1}, \lambda_{2}, p}^{m, n} f(z)=\left(p+\lambda_{2}(k-p)-p \lambda_{1}\right) D_{\lambda_{1}, \lambda_{2}, p}^{m, n} f(z)+\lambda_{1} z\left(D_{\lambda_{1}, \lambda_{2}, p}^{m, n} f(z)\right)^{\prime}
$$

Special cases of this operator include:

- the Ruscheweyh derivative operator in the case $D_{0,0,1}^{0, n} f(z) \equiv R^{n}[6]$,

- the Salagean derivative operator in the case $D_{1,0,1}^{m, 0} f(z) \equiv D^{m} \equiv S^{n}[2]$, $\mathrm{D}_{\lambda_{1}}^{\mathrm{m}}[1]$

- the generalized Salagean derivative operator introduced by Al-Oboudi in the case $D_{\lambda_{1}, 0,1}^{m, 0} f(z) \equiv$

- the generalized Ruscheweyh derivative operator in the case $D_{\lambda_{1}, 0,1}^{1, n} f(z) \equiv D_{n}^{\lambda_{1}}[3]$, and

- the generalized Al-Shaqsi and Darus derivative operator in the case $D_{\lambda_{1}, 0,1}^{m, n} f(z) \equiv D_{n}^{m, \lambda_{1}}[4]$.

To further our work, we need to define a class of functions as follows:

Definition 1.2. A function $\mathrm{f} \in \mathrm{A}_{\mathrm{p}}$ is said to be in the class $\mathrm{S}_{\lambda_{1}, \lambda_{2}, \mathrm{n}}^{\mathrm{m}, \mathrm{j}}[\mathrm{A}, \mathrm{B}, \gamma]$ of $p$-valent functions of complex order $\gamma \neq 0$ in $\mathrm{U}$ if and only if

$$
\left\{1+\frac{1}{\gamma}\left(\frac{z\left(D_{\lambda_{1}, \lambda_{2}, p}^{m} f(z)\right)^{(j+1)}}{\left(D_{\lambda_{1}, \lambda_{2}, p}^{m, n} f(z)\right)^{(j)}}-p+j\right)\right\} \prec \frac{1+A z}{1+B z} .
$$

$\left(z \in \mathrm{U}, \mathrm{p} \in \mathbb{N}, j \in \mathbb{N}_{0}=\mathbb{N} \cup\{0\}, \gamma \in \mathbb{C}-\{0\}, \lambda_{2} \geq \lambda_{1} \geq 0\right)$

Clearly, we have the following relationships:

(i) $\mathrm{S}_{0,0,0}^{0,1,0}[1,-1, \gamma]=\mathrm{S}(\gamma)$ 
(ii) $S_{0,0,0}^{0,1,1}[1,-1, \gamma]=\mathrm{K}(\gamma)$

(iii) $S_{0,0,0}^{0,1,0}[1,-1,1-\alpha]=S^{*}$ for $0<\alpha<1$. The classes $\mathrm{S}(\gamma)$ and $\mathrm{K}(\gamma)$ are said to be classes of starlike and convex of complex order $\gamma \neq 0$ in $\mathrm{U}$ and $\mathrm{S}^{*}(\alpha)$ denote the class of starlike functions of order $\alpha$ in $\mathrm{U}$.

A majorization problem for the class $S(\gamma)$ has been investigated by Altintas e.tal [5] and for the class $S^{*}=S^{*}(0)$ has been investigated by MacGregor [7]. In the present paper, we investigate a majorization problem for the class $S_{\lambda_{1}, \lambda_{2}, \alpha}^{m, p, j}[A, B, \gamma]$.

\section{Majorization problem for the class $S_{\lambda_{1}, \lambda_{2}, n}^{m, p, j}[A, B, \gamma]$}

Theorem 2.1. Let the function $f \in A_{p}$ and suppose that $g \in S_{\lambda_{1}, \lambda_{2}, \mathfrak{n}}^{m, p, j}[A, B, \gamma]$. If $\left(D_{\lambda_{1}, \lambda_{2}, \mathfrak{p}}^{m, n} f(z)\right)^{(j)}$ is majorized by $\left(\mathrm{D}_{\lambda_{1}, \lambda_{2}, \mathrm{p}}^{\mathrm{m}, \mathrm{n}} \mathrm{g}(\boldsymbol{z})\right)^{(\mathrm{j})}$ in $\mathrm{U}$, then

$$
\left|\left(D_{\lambda_{1}, \lambda_{2}, p}^{m+1, n} f(z)\right)^{(j)}\right| \leq\left|\left(D_{\lambda_{1}, \lambda_{2}, p}^{m, n} g(z)\right)^{(j)}\right| \quad \text { for } \quad|z| \leq r_{0},
$$

where $r_{0}=r_{0}\left(p, \gamma, \lambda_{1}, \lambda_{2}, A, B\right)$ is the smallest positive root of the equation

$$
\begin{gathered}
r^{3}\left|\gamma(A-B)-\left(\frac{p+\lambda_{2}(k-p)}{\lambda_{1}}\right) B\right|-\left[\frac{p+\lambda_{2}(k-p)}{\lambda_{1}}+2|B|\right] r^{2}- \\
{\left[\left|\gamma(A-B)-\left(\frac{p+\lambda_{2}(k-p)}{\lambda_{1}}\right) B\right|+2\right] r+\left(\frac{p+\lambda_{2}(k-p)}{\lambda_{1}}\right)=0} \\
(-1 \leq B<A \leq 1 ; P \in \mathbb{N} ; \gamma \in \mathbb{C}-\{0\}) .
\end{gathered}
$$

Proof. Since $g \in S_{\lambda_{1}, \lambda_{2}, n}^{m, p, j}[A, B, \gamma]$ we find from (1.10) that

$$
1+\frac{1}{\gamma}\left(\frac{z\left(D_{\lambda_{1}, \lambda_{2}, p}^{m, n} g(z)\right)^{(j+1)}}{\left(D_{\lambda_{1}, \lambda_{2}, p}^{m, n} g(z)\right)^{(j)}}-p+j\right)=\frac{1+A w(z)}{1+B w(z)}
$$

$(\gamma \in \mathbb{C}-0, j, p \in \mathbb{N}$ and $p>j)$, where $w$ is analytic in $U$ with

$$
w(0)=0 \quad \text { and }|w(z)|<z \quad(z \in \mathrm{U}) .
$$

From (2.3) we get

$$
\frac{z\left(D_{\lambda_{1}, \lambda_{2}, p}^{m, p} g(z)\right)^{(j+1)}}{\left(D_{\lambda_{1}, \lambda_{2}, p}^{m, n} g(z)\right)^{(j)}}=\frac{(p-j)+[\gamma(A-B)+(p-j) B] w(z)}{1+B w(z)}
$$

and

$$
z\left(D_{\lambda_{1}, \lambda_{2}, p}^{m, n} f(z)\right)^{(j+1)}=\left(p+\frac{\lambda_{2}(k-p)}{\lambda_{1}}\right)\left(D_{\lambda_{1}, \lambda_{2}, p}^{m+1, n} f(z)\right)^{(j)}+
$$




$$
\left(p-j-\frac{\lambda_{2}(k-p)}{\lambda_{1}}\right)\left(D_{\lambda_{1}, \lambda_{2}, p}^{m, n} f(z)\right)^{(j)} .
$$

By virtue of (2.4) and (2.5) we get

$$
\left|\left(D_{\lambda_{1}, \lambda_{2}, p}^{m, n} g(z)\right)^{(j)}\right| \leq \frac{\frac{p+\lambda_{2}(k-p)}{\lambda_{1}}[1+|B| z \mid]}{\left(\frac{p+\lambda_{2}(k-p)}{\lambda_{1}}\right)\left|\gamma(A-B)-\left(\frac{p+\lambda_{2}(k-p)}{\lambda_{1}}\right)\right| B|z|}\left|\left(D_{\lambda_{1}, \lambda_{2}, p}^{m+1, n} g(z)\right)^{(j)}\right| .
$$

Next, since $\left(D_{\lambda_{1}, \lambda_{2}, p}^{m, n} f(z)\right)^{(j)}$ is majorized by $\left(D_{\lambda_{1}, \lambda_{2}, p}^{m, n} g(z)\right)^{(j)}$ in the unit disk $U$, we have from (1.2) that

$$
\left(D_{\lambda_{1}, \lambda_{2}, p}^{m, n} f(z)\right)^{(j)}=\varphi(z)\left(D_{\lambda_{1}, \lambda_{2}, p}^{m, n} g(z)\right)^{(j)} .
$$

Differentiating it with respect to $z$ and multiplying by $z$ we get

$$
z\left(D_{\lambda_{1}, \lambda_{2}, p}^{m, n} f(z)\right)^{(j+1)}=z \varphi^{\prime}(z)\left(D_{\lambda_{1}, \lambda_{2}, p}^{m, n} g(z)\right)^{(j)}+z \varphi(z)\left(D_{\lambda_{1}, \lambda_{2}, p}^{m, n} g(z)\right)^{(j+1)} .
$$

Now by using (2.5) in the above equation, it yields

$$
\left(D_{\lambda_{1}, \lambda_{2}, p}^{m, n} f(z)\right)^{(j)}=\frac{z \varphi^{\prime}(z)\left(D_{\lambda_{1}, \lambda_{2}, p}^{m, n} g(z)\right)^{(j)}}{\frac{p+\lambda_{2}(k-p)}{\lambda_{1}}}+\varphi(z)\left(D_{\lambda_{1}, \lambda_{2}, p}^{m, n} g(z)\right)^{(j)}
$$

Thus, by noting that $\varphi \in \Omega$ satisfies the inequality (see, e.g. Nehari [8])

$$
\left|\varphi^{\prime}(z)\right| \leq \frac{1-|\varphi(z)|^{2}}{1-|z|^{2}} \quad(z \in \mathrm{U})
$$

and using (2.6) and (2.8) in (2.7), we get

$$
\begin{gathered}
\left|\left(D_{\lambda_{1}, \lambda_{2}, \mathrm{p}}^{\mathrm{m}+1, n} \mathrm{f}(z)\right)^{(j)}\right| \leq \\
{\left[|\varphi(z)|+\frac{1-|\varphi(z)|^{2}}{1-|z|^{2}} \frac{|z|(1+|\mathrm{B}||z|)}{\frac{p+\lambda_{2}(\mathrm{k}-\mathrm{p})}{\lambda_{1}}-\left|\gamma(\mathrm{A}-\mathrm{B})-\left(\frac{\mathrm{p}+\lambda_{2}(\mathrm{k}-\mathrm{p})}{\lambda_{1}}\right)\right| \mathrm{B} \| z}\right]\left|\left(\mathrm{D}_{\lambda_{1}, \lambda_{2}, \mathfrak{p}}^{\mathrm{m}, \mathfrak{n}} \mathrm{g}(z)\right)^{(j+1)}\right|}
\end{gathered}
$$

which upon setting

$$
|z|=r \quad \text { and } \quad|\varphi(z)|=\rho \quad(0 \leq \rho \leq 1)
$$

leads us to the inequality

$$
\begin{gathered}
\left|\left(D_{\lambda_{1}, \lambda_{2}, p}^{m+1, n} f(z)\right)^{(j)}\right| \leq \\
\frac{\phi(\rho)}{\left(1-r^{2}\right)\left(\frac{p+\lambda_{2}(k-p)}{\lambda_{1}}\right)-\left|\gamma(A-B)-\left(\frac{p+\lambda_{2}(k-p)}{\lambda_{1}}\right) B\right| r}\left|\left(D_{\lambda_{1}, \lambda_{2}, p}^{m+1, n} g(z)\right)^{(j)}\right|
\end{gathered}
$$

where

$$
\begin{gathered}
\phi(\rho)=-r(1+|B|) \rho^{2}+\left(1-r^{2}\right) \\
\left.\left[\left(\frac{p+\lambda_{2}(k-p)}{\lambda_{1}}\right)-\left|\gamma(A-B)+\left(\frac{p+\lambda_{2}(k-p)}{\lambda_{1}}\right) B\right| r\right)\right] \rho+r(1+|B| r)
\end{gathered}
$$


takes its maximum value at $\rho=1$ with $r_{1}=r_{1}\left(p, \gamma, \lambda_{1}, \lambda_{2}, A, B\right)$ for $r_{1}\left(p, \gamma, \lambda_{1}, \lambda_{2}, A, B\right)$ is the smallest positive root of equation (2.2). Furthermore, if $0 \leq \rho \leq r_{1}\left(p, \gamma, \lambda_{1}, \lambda_{2}, A, B\right)$, then function $\psi(\rho)$ defined by

$$
\begin{gathered}
\psi(\rho)=-\sigma(1+|B| \sigma) \rho^{2}+\left(1-\sigma^{2}\right) \\
\left.\left[\left(\frac{p+\lambda_{2}(k-1)}{\lambda_{1}}\right)-\left|\gamma(A-B)+\left(\frac{p+\lambda_{2}(k-p)}{\lambda_{1}}\right) B\right| \sigma\right)\right] \rho+\sigma(1+|B| \sigma)
\end{gathered}
$$

is seen to be an increasing function on the interval $0 \leq \rho \leq 1$ so that

$$
\begin{gathered}
\left.\psi(\rho) \leq \psi(1)=\left(1-\sigma^{2}\right)\left(\frac{p+\lambda_{2}(k-p)}{\lambda_{1}}\right)-\left|\gamma(A-B)+\left(\frac{p+\lambda_{2}(k-p)}{\lambda_{1}}\right) B\right| \sigma\right) \\
0 \leq \rho \leq 1 ;\left(0 \leq \sigma \leq r_{1}\left(p, \gamma, \lambda_{1}, \lambda_{2}, A, B\right)\right) .
\end{gathered}
$$

Hence upon setting $\rho=1$ in (2.13) we conclude that (2.1) of Theorem 2.1 holds true for $|z| \leq$ $r_{1}\left(p, \gamma, \lambda_{1}, \lambda_{2}, A, B\right)$ where $r_{1}\left(p, \gamma, \lambda_{1}, \lambda_{2}, A, B\right)$ is the smallest positive root of equation (2.2). This completes the proof of the Theorem 2.1 .

Setting $p=1, m=0, A=1, B=-1$ and $j=0$ in Theorem 2.1 we get

Corollary 2.1. Let the function $\mathrm{f} \in \mathrm{A}$ be analytic in the open unit disk $\mathrm{U}$ and suppose that $\mathrm{g} \in$ $\mathrm{S}_{0,0,0}^{0,1,0}[1,-1, \gamma]=\mathrm{S}(\gamma)$. If $\mathrm{f}(z)$ is majorized by $\mathrm{g}(z)$ in $\mathrm{U}$, then

$$
\left|f^{\prime}(z)\right| \leq\left|g^{\prime}(z)\right| \quad\left(|z|<r_{3}\right)
$$

where

$$
r_{3}=r_{3}(\gamma)=\frac{3+|2 \gamma-1|-\sqrt{9+2|2 \gamma-1|+|2 \gamma-1|^{2}}}{2|2 \gamma-1|}
$$

This is a known result obtained by Altintas[5].

For $\gamma=1$, the above corollary reduces to the following result:

Corollary 2.2. Let the function $\mathrm{f}(z) \in \mathrm{A}$ be analytic univalent in the open unit disk $\mathrm{U}$ and suppose that $\mathrm{g} \in \mathrm{S}^{*}=\mathrm{S}^{*}(0)$. If $\mathrm{f}$ is majorized by $\mathrm{g}$ in $\mathrm{U}$, then

$$
\left|f^{\prime}(z)\right| \leq\left|g^{\prime}(z)\right| \quad(|z| \leq 2-\sqrt{3})
$$

which is a known result obtained by MacGregor [7].

Some other work related to the class defined by (1.3) can be seen in [9] and of course elsewhere. In fact, recently Ibrahim [10] used the concept of majorization to find solutions of fractional differential equations in the unit disk.

Acknowledgement The work presented here was supported by UKM-ST-06-FRGS0244-2010.

$$
\text { Received: April 2011. Revised: June } 2011 .
$$




\section{References}

[1] F. M. Al-Oboudi, On univalent functions defined by a generalized Salagean operator, Internat. J. Math. Math. Sci., 27(2004), 1429-1436.

[2] G. Salagean, Subclasses of univalent functions, Lecture in Math. Springer Verlag, Berlin, 1013(1983), 362-372.

[3] K. Al-Shaqsi and M. Darus, On univalent functions with respect to $k$-symmetric points defined by a generalization Ruscheweyh derivative operators, Jour. Anal. Appl., 7(2009), 53-61.

[4] M. Darus and K. Al-Shaqsi, Differential Sandwich Theorems with Generalised Derivative Operator, Int. J. Comput. Math. Sci., (22)(2008), 75-78.

[5] O. Altintas, Ö.Özkan and H. M. Srivastava, Majorization by starlike functions of complex order, Complex Var. 46(2001), 207-218.

[6] St. Ruscheweyh, New certain for univalent functions, Proc. Amer.Math. soc. 49(1975),109-115.

[7] T. H. MacGregor, Majorization by univalent functions, Duke Math. J. 34(1967), 95-102.

[8] Z. Nehari, Confformal mapping, MacGraw-Hill Book Company, New York,Toronto and London (1955).

[9] M. Darus and R. W. Ibrahim, Multivalent functions based on a linear operator, Miskolc Mathematical Notes, 11(1) (2010), 43-52.

[10] R. W. Ibrahim, Existence and uniqueness of holomorphic solutions for fractional Cauchy problem, J. Math. Anal. Appl., 380 (2011), 232-240. 